\title{
Pilot study on nocturnal monitoring of crackles in children with pneumonia
}

\author{
Wilfried Nikolaizik ${ }^{1}$, Lisa Wuensch ${ }^{1}$, Monika Bauck ${ }^{1}$, Volker Gross ${ }^{2}$, Keywan Sohrabi ${ }^{2}$, Andreas Weissflog $^{3}$, \\ Olaf Hildebrandt ${ }^{4}$, Ulrich Koehler ${ }^{4}$ and Stefanie Weber ${ }^{1}$
}

${ }^{1}$ Dept of Pediatric Pulmonology, Children's Hospital, Philipps-University, Marburg, Germany. ${ }^{2}$ Faculty of Health Sciences, University of Applied Sciences, Giessen, Germany. ${ }^{3}$ Thora Tech GmbH, Giessen, Germany. ${ }^{4}$ Division of Respiratory and Critical Care Medicine, Philipps-University, Marburg, Germany.

Corresponding author: Wilfried Nikolaizik (wilfried_nikolaizik@hotmail.com)

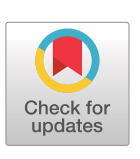

This version is distributed under the terms of the Creative Commons Attribution NonCommercial Licence 4.0. For commercial reproduction rights and permissions contact permissions@ersnet.org

Received: 26 April 2021 Accepted: 9 Sept 2021

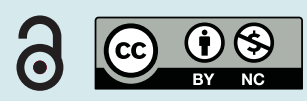

\author{
Shareable abstract (@ERSpublications) \\ Lung sound monitoring can detect crackles in children with pneumonia and might improve \\ diagnosis https://bit.ly/3CfaCl7
}

Cite this article as: Nikolaizik W, Wuensch L, Bauck M, et al. Pilot study on nocturnal monitoring of crackles in children with pneumonia. ERJ Open Res 2021; 7: 00284-2021 [DOI: 10.1183/23120541.002842021].

\section{Abstract}

Background The clinical diagnosis of pneumonia is usually based on crackles at auscultation, but it is not yet clear what kind of crackles are the characteristic features of pneumonia in children. Lung sound monitoring can be used as a "longtime stethoscope". Therefore, it was the aim of this pilot study to use a lung sound monitor system to detect crackles and to differentiate between fine and coarse crackles in children with acute pneumonia. The change of crackles during the course of the disease shall be investigated in a follow-up study.

Patients and methods Crackles were recorded overnight from 22:00 to 06:00 h in 30 children with radiographically confirmed pneumonia. The data for a total of 28800 recorded 30-s epochs were audiovisually analysed for fine and coarse crackles.

Results Fine crackles and coarse crackles were recognised in every patient with pneumonia, but the number of epochs with and without crackles varied widely among the different patients: fine crackles were detected in $40 \pm 22 \%$ (mean \pm SD), coarse crackles in $76 \pm 20 \%$. The predominant localisation of crackles as recorded during overnight monitoring was in accordance with the radiographic infiltrates and the classical auscultation in most patients. The distribution of crackles was fairly equal throughout the night. However, there were time periods without any crackle in the single patients so that the diagnosis of pneumonia might be missed at sporadic auscultation.

Conclusion Nocturnal monitoring can be beneficial to reliably detect fine and coarse crackles in children with pneumonia.

\section{Introduction}

Pneumonia accounts for 16\% of all deaths of children under 5 years old, killing 920136 children in 2015 worldwide [1]. Pneumonia is caused by different infectious agents. The most common bacteria are Streptococcus pneumoniae and Haemophilus influenzae, the most common virus is respiratory syncytial virus [2]. Pneumocystis jirovecii has to be considered in children infected with HIV [3]. Although children are usually not that severely affected, severe acute respiratory syndrome coronavirus 2 (SARS-CoV-2)-associated pneumonia has recently become a worldwide and still ongoing problem $[4,5]$.

Symptoms in children under 5 years of age are not specific and can consist of cough, fever, dyspnoea and lower chest wall indrawing during inspiration [1]. In addition, the diagnosis of pneumonia is based on the results of clinical examination, blood tests and chest radiograph. Owing to the inconsistent features, the diagnosis of pneumonia and the start of appropriate treatment can easily be missed especially in young children.

Specialised monitoring systems can record lung sounds over a certain time and can therefore be considered as a "longtime stethoscope" [6]. The recorded data can be evaluated with a lung sound analysing software. 
The authors have already proven the usefulness of lung sound monitoring in children with obstructive lung disease [7]. To use such systems in children with pneumonia, it is necessary to reliably recognise and characterise crackles in acute pneumonia and during the course of the disease.

The computerised respiratory sound analysis (CORSA) guideline defined fine crackles as a two-cycle duration $<10 \mathrm{~ms}$ and coarse crackles as a two-cycle duration $>10 \mathrm{~ms}$ [8]. Fine crackles indicate pulmonary oedema, pulmonary fibrosis and pneumonia; they are predominantly inspiratory and described as above [9]. Coarse crackles are usually heard at the beginning of expiration and are characteristic of bronchiectasis [9].

Fine crackles might be detected on auscultation in infected areas due to the sudden opening of very small airways during inspiration [10]. In paediatric patients crackles are considered to be fine at the acute stage of pneumonia and become more variable and coarser when the pneumonia is resolving. In infants and toddlers coarse crackles might be the only phenomena based on fluid retention in slightly larger airways. In adults a computerised sound analysis revealed that the crackles in acute pneumonia appear to be coarse and get slightly shorter during recovery [11]. According to the controversial data it is not yet clear what kind of crackles are the characteristic features of acute or resolving pneumonia in children.

It was the aim of this pilot study to record lung sounds in children with acute pneumonia overnight and to analyse the recordings for both fine and coarse crackles. Then, in a follow-up study, the next task shall be to investigate the change of crackles during the further course of the disease.

\section{Methods}

Study subjects

Children admitted to the university hospital in Marburg with respiratory symptoms and suspected pneumonia were eligible to take part in the study. Inclusion criteria were clinical symptoms and infiltrates on the chest radiograph compatible with pneumonia. The Declaration of Helsinki was always followed. The participants and their parents were informed verbally and in writing about the study. Informed written consent was obtained from all participants. The participants were free to withdraw from the study at any time. Approval from the local ethics committee was obtained before the start of the study (Philipps-University Marburg study approval and addendum Az.: 222/12).

\section{Study design}

When the diagnosis of pneumonia had been established, the lung sounds were recorded on the following night from 22.00 to 06.00 h. Every single 30-second epoch per night was audiovisually analysed in all patients for the presence or absence of fine and coarse crackles by an experienced investigator. The data were checked again by a second experienced investigator. Inconclusive data were discussed and finally decided. Cough and wheezing episodes were automatically identified by the lung sound analysing software.

\section{Lung sound monitoring system}

We used the LEOSound monitor (Löwenstein Medical GmbH \& Co. KG, Bad Ems, Germany) to record lung sounds. The LEOSound was originally developed to record lung sounds over a certain time period up to a maximum of $24 \mathrm{~h}$ and to analyse cough and wheezing events automatically.

Three bioacoustic sensors (microphones) will be fixed with adhesive pads dorsally over the right and the left lung and laterally over the trachea. The recording box can be fixed to the patient with a chest belt or lie in the bed beside the patient.

In addition, an ambient microphone is integrated in the LEOSound device. Thus, it is possible to differentiate lung sounds from speech and other ambient sounds. To analyse the data the recording box will be connected with a computer using the lung sound analysing software (Leo-Sound-Analyzer 2.0 Patch 6; Löwenstein Medical GmbH \& Co. KG).

\section{Detection of fine and coarse crackles}

An audiovisual assessment of the recorded lung sounds was performed in every single 30-second epoch of the 30 patients. The American Thoracic Society defined coarse crackles as loud with a low pitch, a two-cycle duration of about $10 \mathrm{~ms}$ and an initial deflection width of about $1.5 \mathrm{~ms}$, while fine crackles are less loud with a higher pitch, a shorter two-cycle duration $(<5 \mathrm{~ms})$ and a shorter initial deflection width (about $0.7 \mathrm{~ms}$ ) [12]. According to these criteria, we determined fine crackles as sharp interrupted high-frequency breath sounds on the audiogram and as equivalent sharp peaks on the spectrogram. In contrast, coarse crackles were louder, had a lower frequency and longer duration, although we did not 
accurately measure the two-cycle duration or the initial deflection width. If the acoustic and visual analysis revealed controversial results, the decision was made in favour of the acoustic recordings. If one or more fine or coarse crackles were detected during a 30-second time period, this epoch was considered to be positive for the equivalent crackle irrespective of the number recorded during this time period.

Analysis

Statistical analysis was performed with GraphPad Prism version 6.0.7 for Windows (GraphPad Software, San Diego, CA, USA; www.graphpad.com).

Results

30 children, eight males and 22 females, were included in the study. The median age was 3.79 years (IQR 4.46, data not normally distributed). The patients already had symptoms for a median of 5.8 days (IQR 5.0) before admission.

Clinical data on admission to the hospital were the following: mean \pm SD temperature $37.9 \pm 1.2^{\circ} \mathrm{C}$, mean $\pm \mathrm{SD}$ breath rate $36.8 \pm 13.8 / \mathrm{min}$, mean \pm SD oxygen saturation measured by pulse oximetry $95.4 \pm 3.1 \%$, median CrP level 41.5 mgL (IQR 90.1, data not normally distributed) and median leukocyte count 13.8 G/L (IQR 10.2).

According to the inclusion criteria all patients had infiltrates on the chest radiograph. Noninvasive microbiological testing consisting of throat swabs and blood serology was positive in 10 of the 30 patients. The most frequently detected pathogen was Mycoplasma pneumoniae in six patients. One patient was positive for respiratory syncytial virus and another for bocavirus. Two patients were positive for multiple pathogens, one for $H$. influenzae and Staphylococcus aureus and another for adenovirus, rhinovirus and bocavirus. 29 patients were considered to have primary bacterial or secondary bacterial pneumonia and were treated with antibiotics including macrolides, cephalosporins and tetracycline. Only one patient was considered to suffer solely from viral pneumonia and did not receive antibiotic treatment. 13 patients received supplemental oxygen during hospitalisation. The median hospitalisation time was 4 days (IQR 1, data not normally distributed). On discharge from hospital antibiotic treatment was continued for a total treatment period of 7-10 days.

Nocturnal rates of crackles and other lung sounds

We analysed the recordings of the 30 patients epoch by epoch throughout the night from 22:00 h to 06:00 h. Altogether, we audiovisually analysed 28800 epochs of $30 \mathrm{~s}$ for fine and coarse crackles in this study. A screenshot of the nocturnal sound recording of a 3.8-year-old boy with left-side paracardial infiltrates on chest radiograph is shown in figure 1.

Fine and coarse crackles were detected in every single patient with pneumonia. However, the crackles were not evident during every single time epoch, and the number of epochs with and without crackles varied widely among the different patients. Audiovisual lung sound analysis revealed that fine crackles were detected in $40 \pm 22 \%$ (mean \pm SD) of the 960 epochs recorded during the night (figure 2). Coarse crackles were detected in $76 \pm 20 \%$. Automated lung sound analysis revealed cough only in $1.5 \%$ (median, IQR 1.8 , data not normally distributed) of the 960 epochs but wheezing in $23 \pm 18 \%$.

Time course of crackles overnight

The time course of epochs with fine or coarse crackles, cough and wheezing was analysed throughout the recording time overnight. The number of epochs with or without fine or coarse crackles varied among the different patients. However, there was no preferential time of the night to detect the different crackles. The distribution of fine and coarse crackle epochs throughout the night was fairly equal and is shown in figure 3. There was no obvious indication that the number of epochs with fine or coarse crackles was related to the clinical condition.

\section{Localisation of crackles}

The side of fine or coarse crackles was localised when the patients underwent a classical auscultation on admission to the hospital. We compared the side of the crackles with the changes on chest radiograph and with the number of crackle epochs on both sides of the lung during overnight monitoring. The detailed results are shown in table 1 .

In 20 of the 30 patients the results of classical auscultation, radiographic changes and the number of total crackle epochs were matching with regard to the side of localisation (67\%). The localisation of radiographic changes was in accordance with the predominant number of total crackle epochs in 21 of the 


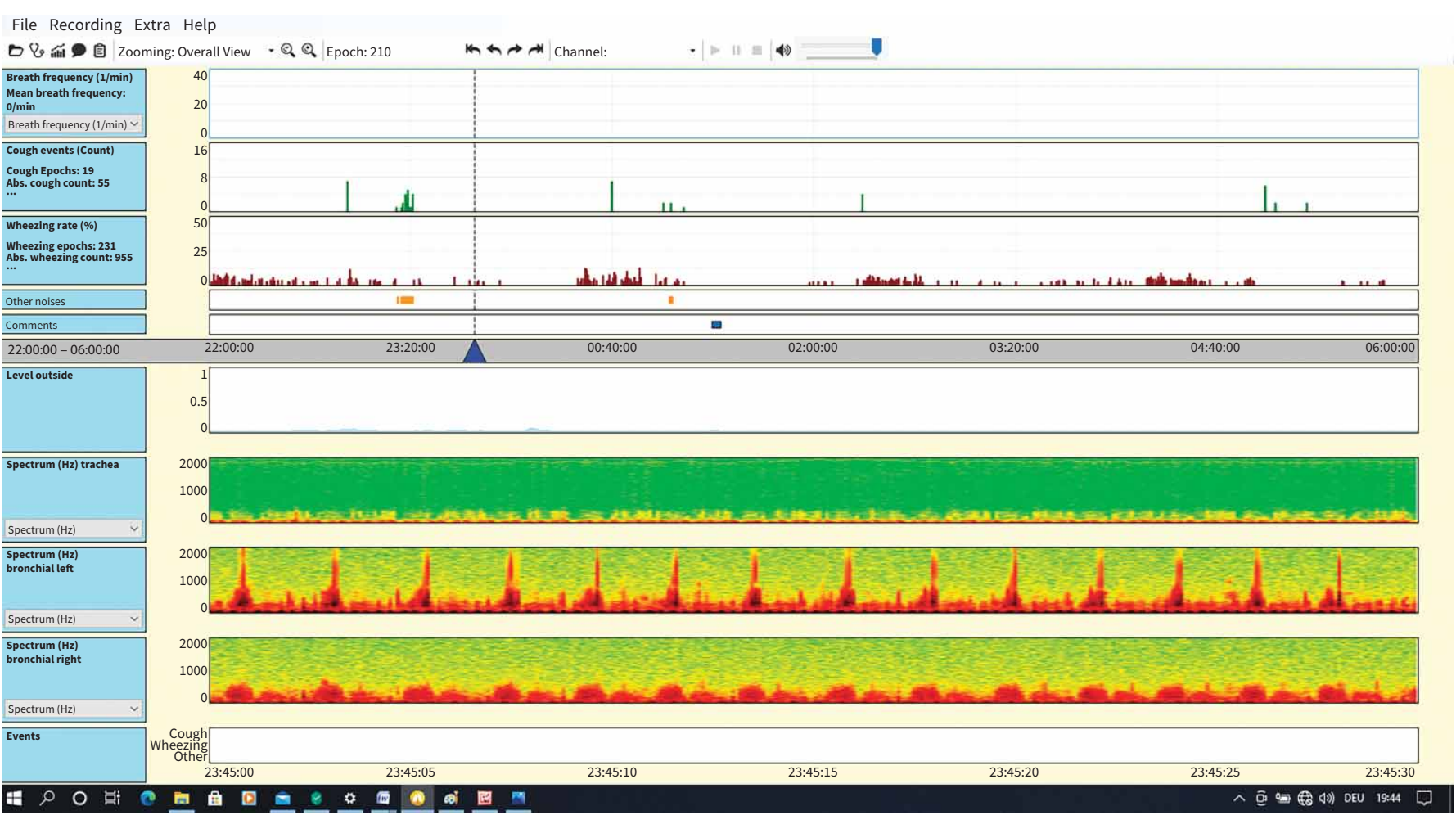

FIGURE 1 Screenshot of the nocturnal lung sound recording of a 3.8-year-old boy with left-sided paracardial infiltrates on the chest radiograph. Microbiological assessment revealed infection with Mycoplasma pneumonia. The leukocyte count was $10.4 \mathrm{G} \cdot \mathrm{L}^{-1}$, the $\mathrm{C}$-reactive protein level $<5 \mathrm{mg} \cdot \mathrm{L}^{-1}$. The graphical user interface consists of a context menu and operating elements in the upper part. The event charts represent an overview of the breath rate in blue, cough events in green, wheezing in red, other sounds in orange and comments in black. Single recording periods can be selected and evaluated with the help of the scroll bar of the corresponding time period shown in grey below. The volume level of the outer microphone is shown in the next bar. The next three segment charts below display the recorded sound spectrum over a 30-second time period separated for trachea, left lung and right lung followed by another segment chart for the recorded events below. The spectrogram at time epoch 210 (23:45:00-23:45:30 h) is showing fine crackles (high-frequency sharp peaks) and coarse crackles (peaks of slightly lower frequency and slightly longer duration) on the chart of the left lung but not on the charts of the right lung or trachea.

30 patients (70\%), with fine crackle epochs in 22 patients (73\%) and with coarse crackle epochs in 23 patients (77\%). In eight patients the classical auscultation on admission had not revealed any crackles (27\%) while fine and coarse crackles were demonstrated in all 30 patients during overnight monitoring. However, when crackles were described and localised on admission, the side coincided with the radiographic changes in 19 of the 20 patients (95\%). The localisation of crackles by classical auscultation was in accordance with the predominant number of total crackle epochs in 16 of the 20 patients (80\%), with fine crackle epochs in 16 patients (80\%) and with coarse crackle epochs in 17 patients (85\%).

\section{Discussion}

Children suffering from pneumonia might be presented with atypical and misleading symptoms [1]. Nowadays, coronavirus disease 2019 is threatening the world, but again children seem to react differently and show milder cases of pneumonia with better prognosis than adults $[4,5]$. Still, severe pneumonia might occur, and an early and reliable diagnosis is essential to make the right diagnosis and prevent permanent lung damage or even death by appropriate treatment [13, 14]. Auscultation of the lung is an important part of the respiratory examination and is helpful in diagnosing various respiratory disorders [15].

In this pilot study we obtained nocturnal lung sound recordings of high quality in 30 patients with pneumonia due to other pathogens than SARS-CoV-2. We analysed the recordings for the presence and distribution of crackles and were able to prove that both fine and coarse crackles were detected in every single patient admitted with acute pneumonia. On some occasions we found an overlap between fine and coarse crackles. So-called medium crackles have already been described and are thought to be caused by air bubbling through mucus in small airways [16]. Assuming that the type of crackles is related to the size 


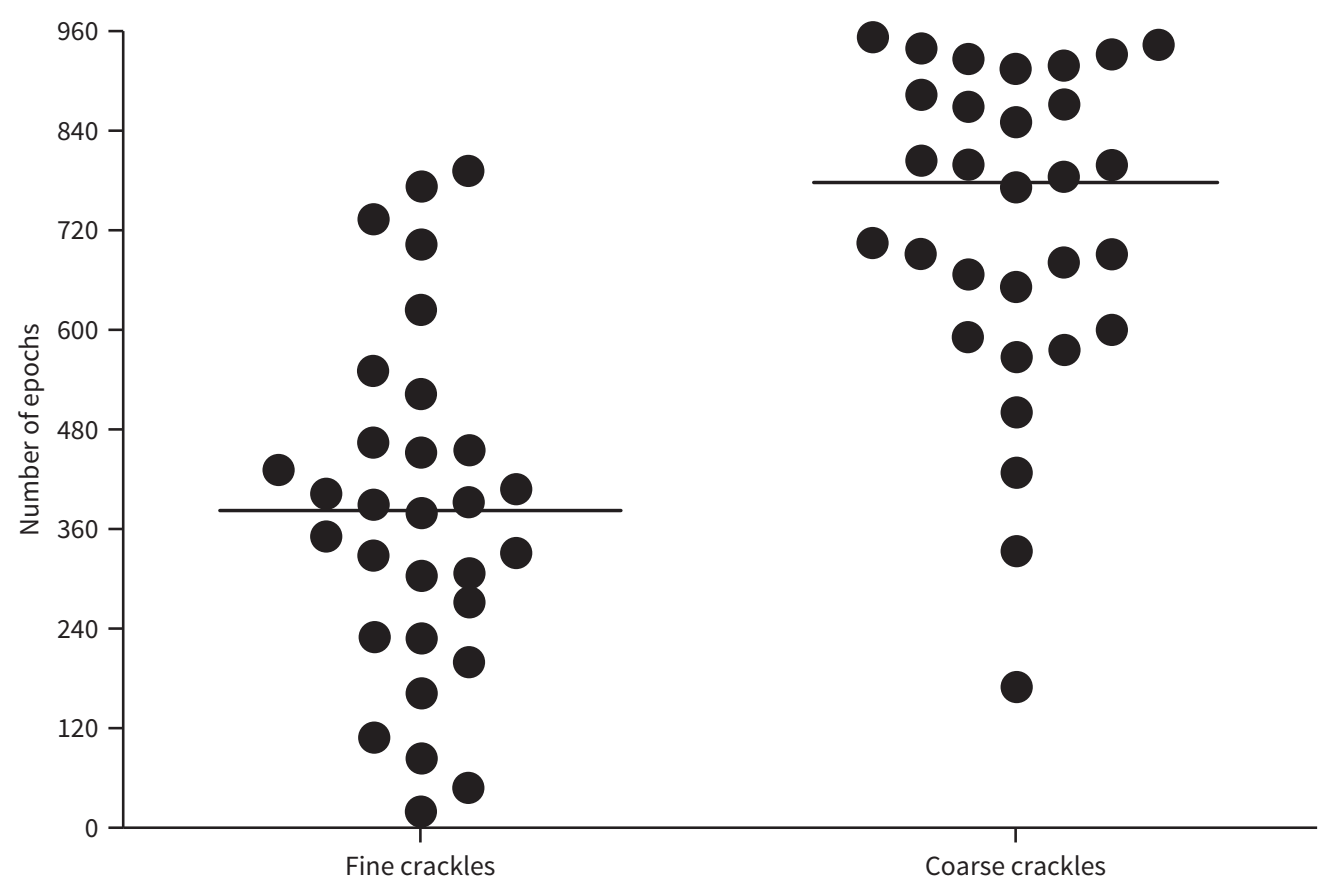

FIGURE 2 Number of epochs with crackles overnight. The number of the 960 recorded epochs (30 s) per patient overnight showing fine and coarse crackles (mean and single patients' results, $n=30$ ).

of the airways [17], then fine crackles are produced within the small airways and coarse crackles arise from larger bronchi [16].

Nowadays, it is not clear what kind of crackles are the characteristic features for acute and resolving pneumonia in children. In clinical practice auscultation reveals fine crackles at least in older children and adults with acute pneumonia [9], while coarse crackles might be the only abnormality in infants and toddlers [18]. In a computerised sound analysis, the crackles in adult patients with acute pneumonia appeared to be mid-inspiratory and fairly coarse with a two-cycle duration of 9 to $11 \mathrm{~ms}$. During recovery the crackles were getting slightly shorter, the two-cycle duration showed a greater variation of 6 to $11 \mathrm{~ms}$, and there was a shift towards the end of inspiration [11]. It was concluded that the crackles in pneumonia resemble those of patients with bronchiectasis in the acute stage and those of patients with fibrosing alveolitis during recovery [19].

It was the aim of this pilot study not only to detect crackles in children with acute pneumonia but also to differentiate between fine and coarse crackles. We have demonstrated that fine crackles were present in $40 \%$ of the epochs but were not detectable in $60 \%$ of the night. Coarse crackles were recorded in $76 \%$, almost twice as many as fine crackles. The number of epochs with and without fine or coarse crackles varied widely among the different patients. In the patient shown in figure 1 fine crackles were recorded in $73 \%$ and coarse crackles in $99 \%$ of the recorded time epochs. In contrast, in another patient (figure not shown) fine crackles were detected only in $2 \%$ and coarse crackles in $18 \%$ of the epochs. This patient had been started on antibiotic treatment already before admission, and pneumonia was probably resolving at the time of admission.

In eight patients no crackles were detected during classical auscultation at admission to hospital. All these patients had fever during hospitalisation and received intravenous antibiotic treatment. According to the inclusion criteria all patients showed infiltrates on the chest radiograph. The CrP level was increased between 21.3 and $278 \mathrm{mg} \cdot \mathrm{L}^{-1}$ in seven of the eight patients. The eighth patient had a normal CrP level of $1.8 \mathrm{mg} \cdot \mathrm{L}^{-1}$ but an increased leukocyte count of $15.0 \mathrm{G} \cdot \mathrm{L}^{-1}$.

Single crackles have also been detected in the breath sounds of normal subjects [20]. Therefore, it is not yet clear how many crackle epochs are required to make a clear diagnosis of acute pneumonia, but normal cut-off ranges might be established in the future. In this pilot study we have proven that both fine and 
Fine crackle epochs

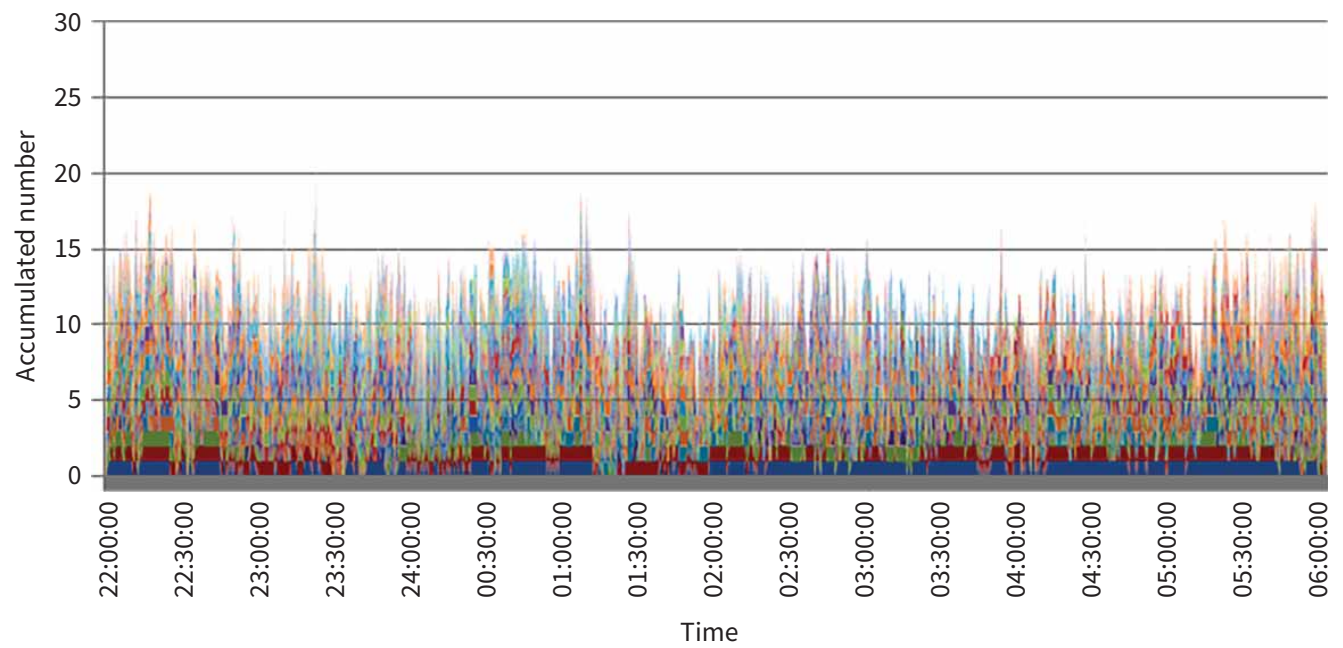

Coarse crackle epochs

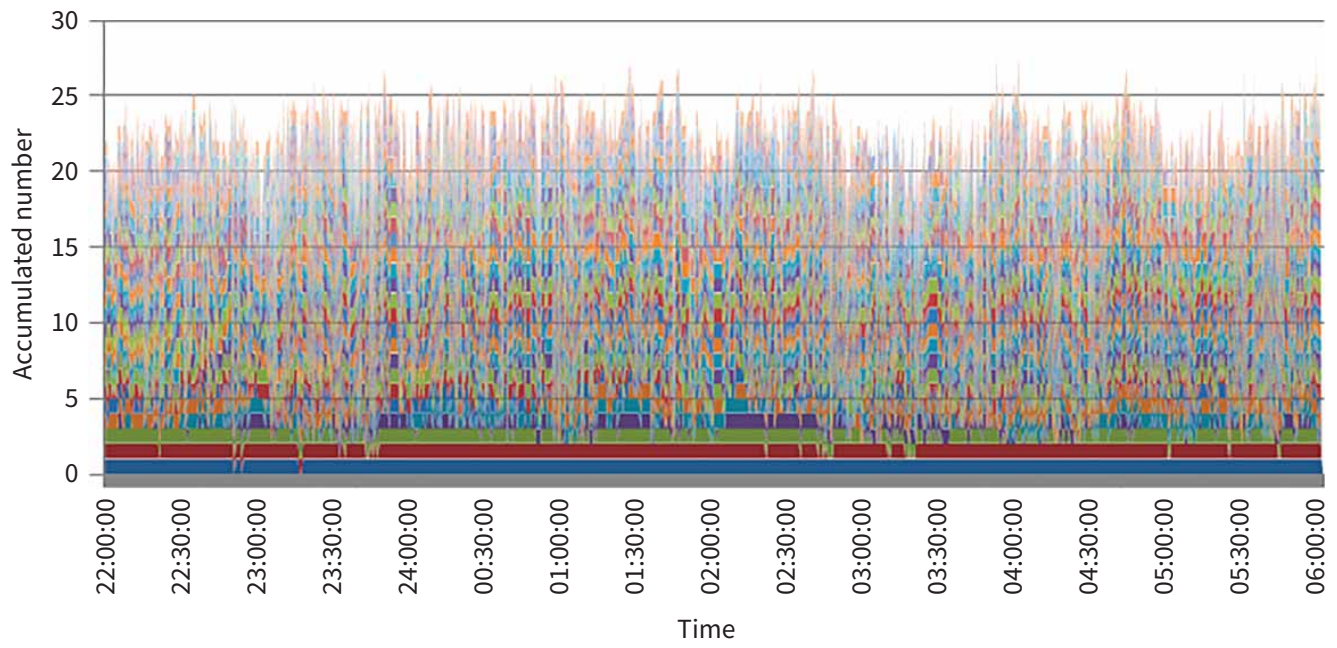

FIGURE 3 Distribution of fine and coarse crackle epochs overnight.

coarse crackles can reliably be detected in children suffering from acute pneumonia. We believe that fine crackles are the characteristic feature of acute pneumonia in children associated with the sudden opening of abnormally closed small airways during inspiration, while coarse crackles indicate the start of resolving pneumonia when secretions congest the slightly larger airways. In this case there should be a shift from mainly fine crackles at acute pneumonia towards mainly coarse crackles during resolving pneumonia. Such further change of crackles during the course of the disease shall be investigated in a follow-up study.

The predominant localisation of crackles as recorded during overnight monitoring was in accordance with the radiographic infiltrates and the classical auscultation in most patients. However, even in patients with radiographic infiltrates solely on one side there were some crackles on the other side too. This might be explained if the sound of crackles can be transferred from one lung to the other. Another explanation might be that a pneumonia is not completely localised to a single region while other lung areas are also infected to some extent. We believe that the latter is more likely especially in children. Furthermore, we have not recorded the sleeping position in this study, which might also have an influence on the presence of crackles. Especially a lateral position leads to a different compression of both lungs and might have an effect on the opening of inflamed airways and the secretions leading to a different crackle pattern. These considerations might explain the discrepancy in the localisation of crackles between overnight monitoring and radiographic changes or classical auscultation in a few patients. 


\begin{tabular}{|c|c|c|c|c|c|c|c|c|}
\hline \multirow[t]{2}{*}{ Patient } & \multirow[t]{2}{*}{ Classical auscultation } & \multirow[t]{2}{*}{ Radiological changes } & \multicolumn{2}{|c|}{ Total crackle epochs } & \multicolumn{2}{|c|}{ Fine crackle epochs } & \multicolumn{2}{|c|}{ Coarse crackle epochs } \\
\hline & & & Left & Right & Left & Right & Left & Right \\
\hline 1 & Left & Left & 1505 & 1404 & 550 & 515 & 955 & 889 \\
\hline 2 & No crackles & Left+right & 1453 & 1407 & 631 & 527 & 822 & 880 \\
\hline 3 & Right & Right & 939 & 1007 & 315 & 345 & 624 & 662 \\
\hline 4 & n.d. & Right & 417 & 582 & 119 & 180 & 298 & 402 \\
\hline 5 & No crackles & Right & 723 & 674 & 271 & 205 & 452 & 469 \\
\hline 6 & Left & Left>right & 1075 & 735 & 467 & 240 & 608 & 495 \\
\hline 7 & No crackles & Right & 840 & 655 & 318 & 258 & 522 & 397 \\
\hline 8 & Left & Right $>$ left & 255 & 785 & 127 & 300 & 128 & 485 \\
\hline 9 & No crackles & Left+right & 1238 & 1246 & 527 & 491 & 711 & 755 \\
\hline 10 & Left & Left>right & 1171 & 799 & 581 & 291 & 590 & 508 \\
\hline 11 & n.d. & Right & 991 & 874 & 283 & 270 & 708 & 604 \\
\hline 12 & Left & Left & 1554 & 1205 & 699 & 392 & 855 & 813 \\
\hline 13 & No crackles & Right & 871 & 1114 & 333 & 280 & 538 & 834 \\
\hline 14 & Left & Left>right & 929 & 951 & 187 & 297 & 742 & 654 \\
\hline 15 & Left+right & Left & 1006 & 1065 & 197 & 144 & 809 & 921 \\
\hline 16 & No crackles & Left>right & 1017 & 777 & 318 & 106 & 699 & 671 \\
\hline 17 & Left+right & Left+right & 1073 & 1096 & 185 & 165 & 888 & 931 \\
\hline 18 & Left+right & Left+right & 106 & 1314 & 46 & 432 & 60 & 882 \\
\hline 19 & Right & Right & 628 & 893 & 182 & 261 & 446 & 632 \\
\hline 20 & Left+right & Right & 1159 & 1052 & 396 & 244 & 763 & 808 \\
\hline 21 & Right & Right & 827 & 1019 & 221 & 313 & 606 & 706 \\
\hline 22 & Right & Left+right & 669 & 764 & 123 & 174 & 546 & 590 \\
\hline 23 & Right>left & Left+right & 1146 & 1318 & 283 & 479 & 863 & 839 \\
\hline 24 & Right $>$ left & Right & 764 & 84 & 75 & 10 & 689 & 74 \\
\hline 25 & Left+right & Right & 831 & 372 & 197 & 29 & 634 & 343 \\
\hline 26 & No crackles & Left & 575 & 466 & 75 & 36 & 500 & 430 \\
\hline 27 & No crackles & Left+right & 184 & 35 & 18 & 2 & 166 & 33 \\
\hline 28 & Right & Right & 422 & 403 & 77 & 84 & 345 & 319 \\
\hline 29 & Left & Left & 281 & 226 & 42 & 20 & 239 & 206 \\
\hline 30 & Left+right & Right & 1041 & 1134 & 219 & 369 & 822 & 765 \\
\hline Mean \pm SD & & & $856.3 \pm 379.4$ & $848.5 \pm 375.4$ & $268.7 \pm 185.9$ & $248.6 \pm 154.5$ & $587.6 \pm 237.0$ & $599.9 \pm 248.3$ \\
\hline
\end{tabular}

Although the distribution of crackles was fairly equal throughout the night, there were time periods without any crackle in every single patient. According to these results, it might be possible to miss the characteristic crackles and the diagnosis of pneumonia if chest auscultation occurs only sporadically. Lung sound monitoring might be helpful to detect or confirm a diagnosis of pneumonia especially in patients with atypical symptoms or long-time hospitalisation. However, such a system needs quick and automated analysis before it is suitable for clinical application. Algorithms for automated analysis of cough and wheezing are already established, and a high sensitivity and specificity has been proven [7]. Such automated analysis to detect crackles and to distinguish between fine and coarse crackles is not yet available for routine use in the lung sound monitoring system we are presenting in this study. For further clinical practice it will be essential to implement such algorithms and to demonstrate a high reliability of the results. Once established, another task will be to investigate the longitudinal course of fine and coarse crackles over consecutive nights and assess if changes of these lung sound recordings come along with clinical changes in the patients. Such association might even help to recognise improvement or deterioration of pneumonia at an early time when the clinical response is not yet apparent.

The application of artificial intelligence has already produced encouraging results in the diagnosis of obstructive lung diseases [21]. An electronic stethoscope with artificial intelligence algorithms was also tested to detect wheezes, rhonchi, and fine and coarse crackles in 50 paediatric patients [22]. The study showed that the automatic sound analysis system was more efficient in detecting all four types of auscultation sounds compared with the results obtained by a group of doctors. Electronic stethoscopes can capture and store lungs sounds in a digital form and generate spectrograms from the recordings. It has also been shown that the use of spectrograms can improve the classification of wheezes and crackles in an 
educational setting [23]. On the other hand, the automatic classification of adventitious respiratory sounds is still an ongoing problem [24]. Currently there are no public databases available to evaluate and compare new algorithms, but the creation and public release of such databases will be useful to solve the respiratory sound classification problem [25].

Acknowledgements: Data from this study were presented at the 25th Jahrestagung Deutsche Gesellschaft für Schlafforschung und Schlafmedizin e.V., Münster, Germany, 9-11 November 2017.

Provenance: Submitted article, peer reviewed.

Conflict of interest: W. Nikolaizik has nothing to disclose. L. Wünsch has nothing to disclose. M. Bauck has nothing to disclose. V. Gross has nothing to disclose. K. Sohrabi has nothing to disclose. A. Weissflog has nothing to disclose. O. Hildebrandt has nothing to disclose. U. Koehler reports grants and personal fees from Löwenstein Medical, outside the submitted work. S. Weber has nothing to disclose.

References

1 World Health Organisation. Pneumonia Fact Sheet. www.who.int/mediacentre/factsheets/fs331/en/ Date last accessed: 6 October 2021. Date last updated: 2 August 2019.

2 El Seify MY, Fouda EM, Ibrahim HM, et al. Microbial etiology of community-acquired pneumonia among infants and children admitted to the pediatric hospital, Ain Shams University. Euro J Microbiol Immunol 2016; 3: 206-214.

3 Saeed NK, Farid E, Jamsheer AE. Prevalence of opportunistic infections in HIV-positive patients in Bahrain: a four-year review (2009-2013). J Infect Dev Ctries 2015; 9: 60-69.

4 She J, Liu L, Liu W. COVID-19 epidemic: disease characteristics in children. J Med Virol 2020; 92: 747-754.

5 Ludvigsson JF. Systematic review of COVID-19 in children shows milder cases and a better prognosis than adults. Acta Paediatr 2020; 109: 1088-1095.

$6 \quad$ Koehler U, Hildebrandt O, Nell C, et al. Akustisches Langzeit-Monitoring von Atem-und Lungengeräuschen im Schlaf. Warum ist das sinnvoll? Somnologie 2014; 18: 225-230.

7 Nikolaizik W. Erste Erfahrungen mit dem LeoSound ${ }^{\circledR}$-Monitor "Langzeitstethoskop" bei Kindern und Jugendlichen mit Atemwegserkrankungen. Schlaf 2016; 5: 27-30.

8 Sovijärvi AR, Dalmasso F, Vanderschoot J, et al. Definition of terms for applications of respiratory sounds. Eur Respir Rev 2000; 10: 597-610.

9 McGee S, ed. Evidence-based physical diagnosis. In: Auscultation of the lungs. Philadelphia, Elsevier Saunders, 2012; pp. 251-266.

10 Koehler U, Hildebrandt $\mathrm{O}$, Kerzel $\mathrm{S}$, et al. Atemgeräusche und Atem-Nebengeräusche. Nomenklatur und visuelle Darstellung. Pneumologie 2016; 70: 1-8.

11 Piirila P. Changes in crackle characteristics during the clinical course of pneumonia. Chest 1992; 102: 176-183.

12 American Thoracic Society. Updated nomenclature for membership reaction. Reports of the ATS-ACCP ad hoc committee. Am Thorac Soc News 1977; 3: 5-6.

13 Rodrigues CMC. Challenges of empirical antibiotic therapy for community-acquired pneumonia in children. Curr Ther Res Clin Exp 2017; 84: e7-e11.

14 Usonis V, Ivaskevicius R, Diez-Domingo J, et al. Comparison between diagnosis and treatment of community-acquired pneumonia in children in various medical centres across Europe with the United States, United Kingdom and the World Health Organization guidelines. Pneumonia 2016; 8: 5.

15 Sarkar M, Madabhavi I, Niranjan N, et al. Auscultation of the respiratory system. Ann Thorac Med 2015; 10: 158-168.

16 Jones A. A brief overview of the analysis of lung sounds. Physiotherapy 1995; 81: 37-42.

17 Crofton J, Douglas A. Common clinical manifestations in respiratory disease. In: Respiratory Diseases, 3rd edition. Oxford, London, Edinburgh: Blackwell Scientific Publications, 1981; pp. 102-108.

18 Pasterkamp H, Kraman SS, Wodicka GR. Respiratory sounds. Advances beyond the stethoscope. Am J Respir Crit Care Med 1997; 156: 3 Pt 1, 974-987.

19 Piirila P, Sovijarvi AR. Crackles: recording, analysis and clinical significance. Eur Respir J 1995; 8: 2139-2148.

20 Workum P, Holford SK, DelBono E, et al. The prevalence and character of crackles (rales) in young women without significant lung disease. Am Rev Respir Dis 1982; 126: 921-923.

21 Das N, Topalovic M, Janssens W. Artificial intelligence in diagnosis of obstructive lung disease: current status and future potential. Curr Opin Pulm Med 2018; 24: 117-123.

22 Grzywalski T, Piecuch M, Szajek M, et al. Practical implementation of artificial intelligence algorithms in pulmonary auscultation examination. Eur J Pediatr 2019; 178: 883-890.

23 Aviles-Solis JC, Storvoll I, Vanbelle S, et al. The use of spectrograms improves the classification of wheezes and crackles in an educational setting. Sci Rep 2020; 10: 8461. 
24 Rocha BM, Pessoa D, Marques A, et al. Automatic classification of adventitious respiratory sounds: a (un) solved problem? Sensors (Basel) 2020; 21: 57.

25 Rocha BM, Filos D, Mendes L, et al. An open access database for the evaluation of respiratory sound classification algorithms. Physiol Meas 2019; 40: 035001. 